\title{
Onomastics of Pirandello's Novels and their Translation into Albanian
}

\author{
Prof. As. Dr. Mirela Papa \\ Docente di Traduzione e Interpretariato, Università di Tirana \\ papamirela@yahoo.com \\ Dr. Alva Dani \\ Docente di Letteeratura italiana, Università di Scutari \\ alvadani2001@yahoo.it
}

\section{Doi:10.5901/mjss.2013.v4n9p650}

\begin{abstract}
Proper nouns are numerous and are found in many books. Man names not only objects and species, but also imagined creatures of a novels. The selection of proper nouns of a literary work is always intentional by the author. The history of proper nouns of Pirandello's characters is characterized not only from the abundance of proper nouns, but also from their originality and meaning. They do not only define novel's character, but they also refer to regions, cities, streets, squares, neighborhoods, lakes, mountains, etc. places where the events in the novel take place. After an initial classification of such nouns into conventional and expressive nouns we would determine the strategies that the Albanian translators have used to translate these nouns categories.
\end{abstract}

Keywords: literature, translation, onomastics, anthroponyms, toponyms.

\section{Introduzione allo studio}

La storia dei nomi propri dei personaggi di Pirandello si presenta come uno degli aspetti più curiosi e significativi del suo lessico. Le novelle di Pirandello abbondano di nomi propri che spiccano per la loro originalità e per il significato che hanno. Su questo aspetto del lessico di Pirandello esistono, pochi studi. Tra quelli possiamo menzionare lo studio di Benvenuto Terracini Considerazioni sullo stile delle novelle di Luigi Pirandello, che si occupa in modo particolare dei nomi e dei cognomi delle novelle di Pirandello. Anche Leonardo Sciascia ha studiato questo tema nel Pirandello dalla $A$ alla Z, pubblicato dall'Espresso in occasione del 50-esimo anniversario della morte dello scrittore. Occorre affermare che le Novelle di Pirandello sono piene di nomi propri, che definiscono e caratterizzano i personaggi, ma si riferiscono anche alle vie, fonti, luoghi ecc. presi dalla realtà o semplicemente inventati. In questo studio intendiamo analizzare i nomi propri usati da Pirandello ed il modo in cui questi nomi arrivano per il lettore albanese tramite le traduzioni.

\section{Onomastica dei personaggi}

I nomi propri ed i cognomi dei personaggi di un'opera letteraria hanno come funzione principale quello di presentare questi personaggi ai lettori. La traduzione dei nomi propri costituisce un punto molto delicato nella teoria e nella pratica della traduzione. Hermans (1985: 13), distingue due categorie di nomi propri partendo dal grado di semantizzazione: conventional names e loaded names. Convenational names sono i nomi a cui manca la carica semantica, sono cioè nomi nella cui composizione non c'è nessuna allusione riguardo alla natura del referente. Loaded names sono i nomi propri semantizzati, dotati di significato o connotazione nella cui composizione troviamo parole che appartengono alle varie parti del discorso.

Per quanto riguarda la traduzione dei nomi propri Franco Aixela (2000: 84-94) divide i procedimenti tecnici in due grandi gruppi: conservazione e sostituzione a seconda del fatto che la traduzione è orientata verso il polo di origine (adeguatezza) oppure verso il polo d'arrivo (accettabilità).

Le strategie traduttive che mirano la conservazione sono: la ripetizione; l'adattamento ortografico; la traduzione linguistica; la glossa extratestuale; la glossa intertestuale.

Appartengono alla categoria delle strategie della sostituzione i seguenti procedimenti: la neutralizzazione limitata; la neutralizzazione assoluta; la naturalizzazione; l'adattamento ideologico; l'omissione; la creazione autonoma. 


\subsection{Conventional names}

Tra i nomi propri dei personaggi delle novelle di Pirandello troviamo ovviamente nomi convenzionali, ossia nomi che non hanno una carica semantica.

Di alcuni personaggi Pirandello cita il nome e il cognome: «Lulù Pulino» Lulu Pulino (L'imbecille); «Silvestro Noli» Silvestro Noli (Notte); «Eleonora Bandi» Eleonora Bandi, «Carlo D'Andrea» Karl d'Andrea, «Giorgio Bandi» Xhorxh Bandi (Scialle nero); «Daniele Castellani» Daniele Kastelani (Un goj) ecc.

Di alcuni altri solo il nome, specialmente quando si tratta di personaggi secondari: «Nerina» Nerina (Filo d'aria) «Gesa» Xheza, «Gerlando» Xherlandi (Scialle nero) ecc. tutti nomi di parenti, familiari, servi ecc.

Bisogna notare anche la passione di Pirandello per i termini che appartengono alle altre lingue. Anche in questo caso si tratta di conventional names.

Nelle sue novelle e racconti il nome straniero di solito è presente in quegli episodi in cui appaiono personaggi stranieri. Per quanto riguarda il trattamento che Pirandello fa di questi esempi di onomastica bisogna sottolineare il fatto che vengono riportati con la loro grafia originale.

Le lingue dalle quali Pirandello ha preso i nomi propri sono: il francese, l'inglese e il tedesco.

Questi nomi appartengono a personaggi episodici, il cui ruolo è secondario nelle novelle: il signor «Charles Trookley» Çarls Trokli, «Miss Ethel Holloway» mis Etel Hallouej, figlia di «Sir W. H. Holloway» Sër U. H. Hallouej, «Baedeker» Bedeker (II capretto nero).

La presenza spagnola nell'onomastica italiana si nota nei cognomi di provenienza spagnola anche se adattati alla fonetica e grafia italiana. Ci riferiamo a «Lopes» Lopes, «Peres» Peres, e «Vignas» Vinjas (Il fumo e Lo scaldino).

La strategia seguita da parte dei traduttori albanesi per la traduzione dei nomi propri, sia italiani che stranieri, è la stessa per quanto riguarda i conventional names: l'adattamento ortografico. Questa strategia orienta la traduzione verso il polo d'origine.

\subsection{Loaded names}

Pirandello a volte sceglie i nomi dei suoi personaggi incongruenti ed esagerati, altre volte volgari e mediocri. Occorre dire che caratteristica di quasi tutti i nomi dei personaggi di Pirandello è che sono nomi parlanti, che denotano, cioè, le caratteristiche del personaggio. Molti personaggi vengono menzionati con un soprannome. Molti di questi nomignoli alludono all'aspetto fisico del personaggio altri, però, caratterizzano le loro passioni, professioni, tendenze ecc.:

- Carlandrea Sciaramé (...) e la chiamavano la Garibaldina. (Le medaglie)

- Karlandrea Sharame (...) dhe e thërritnin Garibaldinasja. (Medaljet, p. 7, «Zoja prej dylli», Toena, Tiranë 2010)

- Cesare, il Milanese, come non sai? (Lo scaldino).

- Se s'e ditke, Cezare Milanezi! (Mangalli, p. 46, «Zoja prej dylli», Toena, Tiranë 2010)

Ci sono addirittura novelle che portano nel titolo i soprannomi destinati ai protagonisti. Rondone e Rondinella:

- Meglio chiamarli Rondone e Rondinella, come tutti li chiamavano in quel paesello di montagna: Rondone e Rondinella, non solo perché ritornavano ogni anno, d'estate, non si sa donde, al vecchio nido; non solo perché andavano, o meglio, svolavano irrequieti dalla mattina alla sera per tutto il tempo che durava il loro soggiorno colà; ma anche per un'altra ragione un po' meno poetica.

- Mâ mirë me i quejt Dallandyshi e Dallandyshja, sikurse i quejshin të gjithë ata që ishin n'at katund: Dallandyshi e Dallandysheja, por jo vetëm sepse ktheheshin ç'do verë n'at vend në çerdhen e tyne, e sepse gjatë kohës që rrijshin aty shëtitshin të gëzuem e të ç'kujdesun për ç'do gjâ, por edhe për një arësye tjetër, që âsht ma pak poetike. («Tomori», 1941, 12 giugno)

La ragione di questo nomignolo si trova nella novella stessa:

- E vedendolo andar così, con quel farsetto nero e quei calzoni bianchi, come non chiamarlo Rondone?

- E tue pa që kalonte kështu me at pallto të zezë, me pantallone të bardhë, si mos me e quejt Dallandysh? («Tomori»), 1941, 12 giugno)

A volte il significato ironico del nome-etichetta è molto evidente:

- Con questo mio amico, che ha un nome curiosissimo, non si scherza, bada! - Antropofago Capribarbicornipiede! - esclamò l'altro con un versaccio. (Amicissimi)

- Kij kujdes se s'bëhet shaka me këtë shokun tim që ka një emër fort të çuditshëm. - Antropofag Mjekërbrikëmbëcjapi! - ia pat tjetri. (Shokë të ngushtë, p. 70, «Nata e parë», Toena, Tiranë 2008)

Come possiamo notare negli esempi riportati i traduttori albanesi hanno seguito la strategia della traduzione 
linguistica per questi loaded names. Questa è una strategia che orienta la traduzione verso il polo di origine e che rispetta l'intentio auctoris.

\subsection{Nomi propri e i giochi di parole}

A Pirandello piace giocare con l'onomastica, che la fa diventare oggetto di molteplici giochi fonetici. I nomi propri delle persone soffrono di alterazioni grafiche e fonetiche nella bocca degli altri personaggi, e qui si evidenzia ancor una volta la scarsa importanza del nome come rappresentanza e simbolo della persona nominata. (Blanco 1990: 317) Così, nella novella Marsina stretta il professor Gori ha difficoltà a ricordare il nome dell'altro personaggio:

- Signor Grimi...

- Migri, prego.

- Ah già, Migri... (...)! Dicevo Grimi, Mitri, Griti... (Marsina stretta)

- Zoti Grimi...

- Ju lutem, Migri.

- Ah, po, Migri...! Thosha me vete Grimi, Mitri, Griti... (Fraku i ngushtë, p. 166, «Nata e parë», Toena, Tiranë 2008)

Nella novella L'altro figlio la vecchia Maragrazia, rimbecillita dalla mancanza di notizie di suo figlio emigrato in America, chiede al medico di famiglia:

- Ha sentito parlare vossignorìa d'un certo Canebardo? - Garibaldi? - (...) Sissignore

- Kini dëgjuar zotria juaj të flitet për një farë Kanebardo? - Garibaldi? - Po, po, vazhdoni! (Djali tjetër, p. 163, «Jeta lakuriq», Naim Frashëri, Tiranë 1963)

In alcuni casi il nomignolo serve per prendere in giro:

- Ehi, Dorina, su! — chiamò il cameriere. — Vedi che c'è qui il signor Bonvicino.

- Bonavino, - corresse Micuccio, che stava a soffiarsi su le dita.

- Bonavino, Bonavino, conoscente della signora. (Lumie di Sicilia)

- Çohu, Dorinë! - tha kamarieri. - S'e sheh që ka ardhur zoti Bonviçino)

- Bonavino, - e korrigjoi Mikuço, i cili po u hukaste gishtave.

- Bonavino, Bonavino, një i njohur i zonjës. (Limona Sicilie, p. 162, «Tregime e novela», Dituria, Tiranë 1999)

Negli ultimi due casi si tratta di nomi convenzionali che possono avere un senso etimologico. Canebardo (cane + bardo), Bonvicino (bon + vicino). La strategia seguita dai traduttori per la traduzione di questi nomi è l'adattamento ortografico. Con questa strategia si perde ovviamente il gioco semantico.

- mi chiama Pitagora perché non mangio fagiuoli; e spiegato anche perché, a mo' d'ingiuria scherzosa, si può chiamar Pitagora chi non mangi fagiuoli (La disdetta di Pitagora)

- më quan Pitagora, për shkak se nuk i ha fasulet; ai do t'i ketë shpjeguar edhe se përse quhet, me të qeshur, Pitagora kush nuk ha fasule (Tersllëku i Pitagorës, p. 78, «Jeta lakuriq», Çabej, Tiranë 1997)

A volte la presa in giro risalta quando il soprannome viene seguito dalla spiegazione:

- (...) don Marcuccio La Vela conosciuto con... il nomignolo di Cirlinciò, che in Sicilia, per chi volesse saperlo è il nome di un uccello sciocco" (La berretta di Padova)

- don Markuçjo La Vela... nofkës Çirlinçjò, që në Sicili, për ata që duan ta mësojnë, quajnë një zog torollak. (Kësula padoviane, p. 92, «Teze Mikelina», Toena, Tiranë 2010)

La spiegazione che segue il soprannome fa sì che l'intenzione dell'autore venga trasmessa anche al lettore della traduzione.

\subsection{Altre caratteristiche dell'onomastica dei personaggi}

In molti casi Pirandello si serve di altri procedimenti per la creazione dei nomi. Tra questi procedimenti possiamo menzionare la suffissazione, l'aferesi, l'apocope e la composizione. È un grande pregio dell'italiano l'avere i modi per esprimere con una sola parola la sostanza o la qualità accompagnate dalle idee accessorie di grossezza, piccolezza, graziosità, viltà, e malvagità; adoperando gli accrescitivi, i diminutivi, gli spregiativi, i peggiorativi. Tutto il contrario avviene con la lingua albanese. Nell'albanese standard questo fenomeno non è frequente, anzi formazioni simili di solito non vengono dati nei dizionari, perché non trovano spazio come le altre parole. (Lloshi 2001: 119).

I suffissi più usati da Pirandello sono - ino e - etto: «Teresina» Terezina (Lumie di Sicilia); «Titina» Titina (Come gemelle) ecc. 
Tra gli altri suffissi vezzeggiativi possiamo menzionare: - uccio e - uzzo: «Milluccia» Miluçja (Come gemelle), «Guiduccio» Guiduçjo (La madonnina), «Januzza» Janucka (ll fumo), «Micuccio» Mikuço (Lumìe di Sicilia).

Non essendo frequente questo fenomeno nell'albanese i traduttori hanno semplicemente seguito la stessa strategia seguita per gli altri nomi convenzionali, cioè l'adattamento ortografico. Solo nel caso del nome «Januzza» il traduttore ha usato nella traduzione un suffisso vezzeggiativo tipico di una regione del Sud-Est d'Albania. Qui abbiamo a che fare con una perdita nel processo di traduzione, perché non viene rispettata l'intenzione dello scrittore.

L'apocope è un fenomeno che si usa nel linguaggio popolare. Costituisce ovviamente una caratteristica dialettale. Alcuni esempi di questo fenomeno: «Mamm'Antò Nëna Antò (Prima notte); Nunziata «Nunzià» Nuncià (Prima notte); Marastella «Marastè» Marasté (Prima notte); Nocio Butera «Don No» don Noçio (Il fumo).

I traduttori hanno adattato ortograficamente i nomi. Si può intuire questa caratteristica della lingua italiana anche se è un fenomeno che non ricorre nella lingua albanese.

L'aferesi gioca un ruolo meno importante nella creazione dei nomi propri di persone (consiste nella perdita di un suono o gruppo di suoni all'inizio di una parola). Alcuni esempi: «Milio» Milio (don Camillo, Come gemelle); «Nino» Nino (Antonino, Il ventaglino); «Duccio» Duçjo (Guiduccio, La madonnina).

L'adattamento ortografico da parte dei traduttori permette al lettore albanese di intuire questo fenomeno che è presente anche nella sua lingua.

Le forme don-donna sono molto usate davanti ai nomi propri. Don si usa di solito come titolo di rispetto per i sacerdoti, oppure precede i nomi propri maschili di un certo rango elevato: «un santo sacerdote, don Lagaipa» don Lagaipa (Il tabernacolo), «il sacerdote don Arturo Filomarino» prifti don Arturo Filomarino (Fortunati) ecc.

Occorre aggiungere però che nel sud d'Italia la forma don può precedere anche altri nomi di origine modesta, $\mathrm{e}$ dicono, per esempio, salutando il portinaio, Buongiorno, don Ciccillo, ma si tratta di un uso che risale al periodo della dominazione spagnola; per cui il don Ciccillo di Napoli corrisponde esattamente al signor Francesco di Firenze 0 di Milano (Gabrielli 1997: 16).

Alcuni esempi: «don Lisi Chirico» don Lizi Kiriko (Prima notte), «don Mattia Scala» don Matia Skala (II tabernacolo), «don Antonio Sclepis» don Antonio Sklepia (Difesa di Mèola), «don Lollò Zirafa» don Lolò Xirafa (La giara) ecc.

La forma femminile donna mantiene il primo significato di signora, donna di casa, anche se si può usare per esprimere cortesia e rispetto: «donna Nela» dona Nela (Prima notte) ecc.

La strategia seguita dai traduttori è la ripetizione di queste forme nel testo tradotto. Si tratta di una strategia orientata verso il polo di origine. II lettore albanese capisce che si tratta di un termine tipicamente italiano senza poter fare la distinzione quando si usa come titolo di rispetto e quando invece viene usato senza questa intenzione. In questo caso occorre dire che le differenze culturali provocano perdite nel processo traduttivo.

\section{Toponimia}

In tutta la produzione delle novelle di Pirandello si avverte sempre la provincia italiana. Le azioni umane, i fatti, le circostanze dimostrano di essere stati tratti da un fondo di esperienze girgentine, o per lo meno, palermitane, conservate nella memoria di Pirandello. La maggioranza dei suoi racconti può avere una localizzazione reale nella geografia italiana. Il lettore sa dove si svolgono le vicende non perché Pirandello propone i dati, ma dal contenuto reale del racconto: urbano o cittadino, Sicilia o Roma.

In alcuni casi la toponimia è chiara:

- Miss Ethel Holloway, (...), capitò in Sicilia a Girgenti, per visitarvi i meravigliosi avanzi dell'antica città dorica. (II capretto nero)

- mis Etel Hallouej, (...), shkoi në Sicili, në Xhirxhenti, për të vizituar rrënojat e famshme të qytetit të lashtë dorik. (Keci i zi, p. 148, «Tregime e novela», Dituria, Tiranë 1999)

Micuccio nella novella Lumie di Sicilia, «veniva dalla provincia di Messina» Mesina e Teresina «a Napoli, bisognava mandarla al conservatorio di Napoli a qualunque costo» Napoli (Lumie di Sicilia), La giara si svolge in «Sicilia» Sicili, Acqua e li in Milocca, L'imbecille a Costanova Kostanova ecc.

I nomi di vie, fonti, industrie, commerci ecc. che figurano nei suoi racconti, presi dalla realtà o inventati, presentano alcuni casi di grande originalità.

«La Angiolina» Anxholina, «Colomba» Pëllumbesha, «Fratelli Noghera» Vëllezërit Nogera, «Annunziatella» Anunciatela (nomi di barche nella novella La maestrina Boccarme); «il tempio della Concordia» tempullit të Harmonisë, «quello di Hera Lacinia», ai i Hera Lacinia «quell'altro detto volgarmente dei Giganti» apo të atij që populli e quan 
Tempulli i Gjigandëve (Il capretto nero); «Bosco della Cìvita» pylli i Çivitas (il Capretto nero) ecc.

La strategia dei traduttori in questo caso è l'adattamento fonetico per i conventional names e la traduzione per i loaded names. Così «Colomba», «il tempio della Concordia», «il tempio dei Giganti» vengono tradotti nell'albanese.

Da notare che Pirandello mette dei nomi propri anche ai vari animali che popolano i suoi racconti (cani, gatti, passeri, galli, galline, cavalli, scimmie ecc.)

Esempi:

- Tè, Scampirro! Tè, Regina! (nomi di cani nella novella II fumo),

- Hej, na Skampir! Hej, na Rexhinë! (Tymi, p. 55, «Jeta lakuriq», Naim Frashëri, Tiranë 1963)

- La scimmia si chiamava Tita; era vecchia e tisica per giunta. (II fumo)

- Majmunka quhej Tita; ish plakë e për më tepër tuberkuloze. (Tymi, p. 66, «Jeta lakuriq», Naim Frashëri, Tiranë 1963)

- «Fofo» Fofoi, «Nero» Karaj, «Corbino» Korbin (cavalli nella novella La rallegrata) ecc.

Nel caso dei nomi degli animali «Regina» non viene tradotto, anche se si tratta di un nome semantizzato, mentre «Nero» il nome del cavallo è stato tradotto in albanese. Gli altri nomi, essendo nomi convenzionali vengono solo adattati alle regole fonetiche-morfologiche della lingua.

\section{Conclusioni}

In conclusione di questo studio possiamo dire che la strategia principale per la traduzione dei conventional names è l'adattamento ortografico. Per i loaded names abbiamo nella maggioranza dei casi traduzione dei nomi propri. Teoricamente i nomi delle persone e gli oggetti sono esterni alla lingua, non hanno un senso e non devono essere tradotti. Però visto che i nomi propri dei personaggi di Pirandello sono nomi «self-interpreting» e servono per caratterizzare meglio il personaggio che designano, sarebbe allora meglio che la strategia adottata da parte dei traduttori fosse la traduzione dei nomi dotati di una carica semantica oppure l'introduzione di una glossa extratestuale per tradurre il loro significato.

Per gli altri nomi propri, ossia i toponimi la strategia principale è sempre quella dell'adattamento ortografico per i conventional names e a volte la traduzione per i loaded names.

I giochi fonetici e semantici voluti dall'autore non possono ovviamente essere riproposti per i lettori della traduzione visto che dipendono dalle particolarità delle lingue.

In definitiva possiamo dire che si notano delle perdite nel processo traduttivo dei nomi propri delle novelle di Pirandello, perdite dovute alle peculiarità culturali e linguistiche.

\section{References}

Pirandello, L. (1963). Jeta lakuriq (La vita nuda), Tirana: Naim Frashëri. , (1997). Novela për një vit. Jeta lakuriq (La vita nuda), Tirana: Çabej. (1999), Tregime e Novela (Racconti e novelle), Tirana: Dituria. (2008). Nata e parë (Prima notte), Tirana: Toena. (2008). Zoja prej dylli (La Madonnina), Tirana: Toena. (2010). Teze Mikelina (Zia Michelina), Tirana: Toena.

Blanco, A. N. (1990). La lengua y el estilo di Luigi Pirandello, tesis doctoral.

Franco Aixela, J. (1996). Culture-specific items in Translation, në Román Alvarez dhe Ma Carmen Africa Vidal, Translation, Power, Subversion, Clevedon: Multilingual Matters.

Gabrielli, A. (1997). Nella foresta del vocabolario, Milano: Mondadori.

Hoxha, G. Fjalët italishte në shqipe, «Rilindja», 13 mars 1976.

Hermans, Th. (1985) Translation Studies and a New Paradigm, tek Theo Hermans (ed.) The Manipulation of Literature: Studies in Literary Translation, London: Croom Helm, p. 7-15.

Lloshi, Xh. (2001). Stilistika e gjuhës shqipe dhe pragmatika, Tirana: shblu.

Newmark, P. (1981) Approaches to Translation, Oxford: Pergamon Press.

, (1987) A textbook of translation, London: Prentice Hall.

Papa, M. (2012). Translating figurative images of II fu Mattia Pascal, in «Mediterranean Journal of Social Sciences» Vol. 3 N. 2 , publisher Mediterranean Center of Social and Educational Research, fq. 497-506, Roma, Italia, ISSN 2039-9340.

Terracini, B. (1982). Analisi Stilistica, Teoria, storia e problemi, Milano: Feltrinelli.

Torre, E. (1994). Teoria de la traducciòn literaria, Madrid: Sìntesis. 